Abstract ID: 167

Clinical

POSTER

\title{
Comparison of Blood Loss using Intra-Articular Injection of Tranexamic Acid after Total Knee Replacement
}

Aziz Sabbir Husain

Department of Orthopaedic, Traumatology and Rehabilitation, International Islamic University Malaysia, Kuantan Pahang.

Introduction: Total knee replacement (TKR) is one of the most common surgeries in orthopedic field. Up to $1 / 3$ of the patients require blood transfusion postoperative. Allogenic transfusion has many side effects. Tranexamic acid (TXA) is a synthetic antifibrinolytic agent. We explore the usage of TXA in reducing blood loss and transfusion rate by injecting it into the knee joint during surgery. Materials and method: This is a retrospective study done in Hospital Tengku Ampuan Rahimah, Klang. Medical records of patient undergoing TKR between 1 January 2018 till 31 December 2018 were reviewed. Study sample was calculated as 90 patients and divided into 2 groups, one receiving TXA (study) and the other not receiving TXA (control). Post-operative hemoglobin levels and transfusion rate was recorded. Results: 45 patients in study group (17 male, 28 female) had a mean age of 65.4 years old and 45 patients in control group ( 24 male, 21 female) had a mean age of 64.2 years old. Mean post-operative hemoglobin drop in study group was $1.08 \mathrm{~g} / \mathrm{dL}$ vs $1.86 \mathrm{~g} / \mathrm{dL}$ in control group. Repeated measure ANOVA determined a $\mathrm{p}$ value of 0.001 which shows a significant correlation. Total transfusion rate in study group was 1 vs 9 in the control group. Using chi-square test, the $p$-value was 0.007 which again shows a statistically significant result. Conclusion: Intra-articular injection of TXA following TKR reduces blood loss and the need for blood transfusion without increasing any complication. This prevents many patients from undergoing a potentially hazardous blood transfusion. A routine usage of intra-articular TXA in patients undergoing TKR is recommended. However a large and well designed RCT is required to investigate the risk and benefits of TXA. 